\title{
Das Statin-Conundrum (zum Zweiten)
}

\author{
Nikola Biller-Andorno ${ }^{a}$, Stefan Felder ${ }^{a}$, Christoph A. Meier ${ }^{b}$, Urs Metzgera, Brigitte Taga \\ a Für den Expertenrat des Swiss Medical Board (SMB) \\ b Fachspezialist für den SMB-Bericht über «Statine zur Primärprävention kardiovaskulärer Erkrankungen» vom 30.11.2013
}

Über den Statinbericht des Swiss Medical Board (SMB) haben wir in der Schweizerischen Ärztezeitung mit den Autoren Romanens et al. unter dem Titel «Das StatinConundrum» schon einmal die Klingen gekreuzt [1, 2]. In diesem Bericht wird die Frage bearbeitet, ob bei Personen, die keine diagnostizierte kardiovaskuläre Erkrankung aufweisen (sogenannte «Primärprävention»), eine Verschreibung von Statinen mit einem relevanten Vorteil bezüglich Anzahl möglicher künftiger kardiovaskulärer Ereignisse, der Überlebensdauer und der Lebensqualität verbunden ist. Die Empfehlungen lauten wie folgt:

- Eine Verschreibung von Statinen zur Primärprävention soll erst in Betracht gezogen werden, wenn die anderen Möglichkeiten zur Reduktion der Risikofaktoren ausgeschöpft sind.

- Das Risiko für ein kardiovaskuläres Ereignis muss individuell gemäss den gültigen Guidelines bestimmt werden (AGLA, Arbeitsgruppe Lipide und Atherosklerose der Schweiz. Gesellschaft für Kardiologie, ESC, European Society of Cardiology).

- Eine Verschreibung von Statinen in der Primärprävention ist bei einem Risiko für ein tödliches kardiovaskuläres Ereignis (innerhalb von 10 Jahren) von unter 7,5\% (gemäss ESC-Score) nicht indiziert.

Streitpunkt ist die Höhe dieses Risikos, ab dem eine Statintherapie zur Primärprävention indiziert ist. Offenbar sind Romanens et al. mit unseren gesundheitsökonomischen Berechnungen nicht zufrieden und stellen fest: "Als Kontrahenten haben wir bei den HTAs (Health Technology Assessments) einerseits die naturwissenschaftlich arbeitende Medizin, deren Erkenntnisse und Fortschritte aus der Aufarbeitung konkreter Einzelfälle resultieren, und andererseits die Gesundheitsökonomie, welche die Sorge um die Bezahlbarkeit dieser Fortschritte dazu bringt, die Erkenntnisse der Medizin vermehrt in Frage zu stellen.» Sie konstruieren damit einen vermeintlichen Gegensatz zwischen Medizin und Ökonomie. Das Gegenteil ist richtig: Ein Mediziner, der die Zweckmässigkeit und die Wirtschaftlichkeit nicht beachtet, ist ein schlechter Mediziner. So ist eine unnötige Verschrei- bung unethisch, weil die dafür aufgewendeten Ressourcen anderen Patienten für eine wirksamere Intervention zugutekommen könnten. Auch sollte eine teurere, aber vom Ergebnis her nicht bessere Intervention unterbleiben, weil sie ebenfalls zu einer inadäquaten und für die Bevölkerung nicht zu rechtfertigenden Ressourcenverschwendung führt. Darum geht es bei der Unternehmung SMB: Empfehlungen abzugeben über medizinische Interventionen, die mehr schaden als nützen, oder Anpassungen in der Indikationsstellung vorzuschlagen, wie dies beim Statinbericht geschehen ist. Das Ziel ist hierbei einzig und allein, den Menschen unseres Landes mit den zur Verfügung stehenden Mitteln die bestmögliche medizinische Versorgung zu garantieren entsprechend den gesetzlichen Vorgaben (KVG Art. 32, WZW-Kriterien).

Kosten von 210000 CHF pro qualitätsbereinigtes Lebensjahr (QALY) für die Verschreibung von Statinen beim Schwellenwert eines 5\%-Risikos, in den nächsten zehn Jahren an einem kardiovaskulären Ereignis zu versterben, erscheinen dem SMB im Vergleich zu anderen mit diesen Ressourcen möglichen Interventionen als zu hoch. Es hat deshalb empfohlen, den Schwellenwert auf 7,5\% zu erhöhen, ab dem eine Verschreibung von Statinen zur Primärprävention in Betracht gezogen werden soll.

Wie ist das SMB auf diese Zahl gekommen? Wie Romanens et al. richtig schreiben, ist das SMB für Patienten, deren Risiko beim heute verwendeten Schwellenwert

Eine unnötige Verschreibung ist unethisch, weil die dafür aufgewendeten Ressourcen anderen Patienten zugutekommen könnten.

von 5\% liegt, davon ausgegangen, dass eine prophylaktische Statinbehandlung von 1000 Patienten über fünf Jahre zwei Todesfälle und neun Herzinfarkte oder Schlaganfälle vermeidet. Diese elf vermiedenen Fälle sparen insgesamt Kosten von 350000 CHF oder 350 CHF pro behandelte Person ein. Die Kosten einer fünfjährigen Statinbehandlung betragen $2350 \mathrm{CHF}$; netto entstehen somit Kosten von 2000 CHF pro behandelte Person. Dem steht ein Nutzengewinn von 0,0095 quali- 


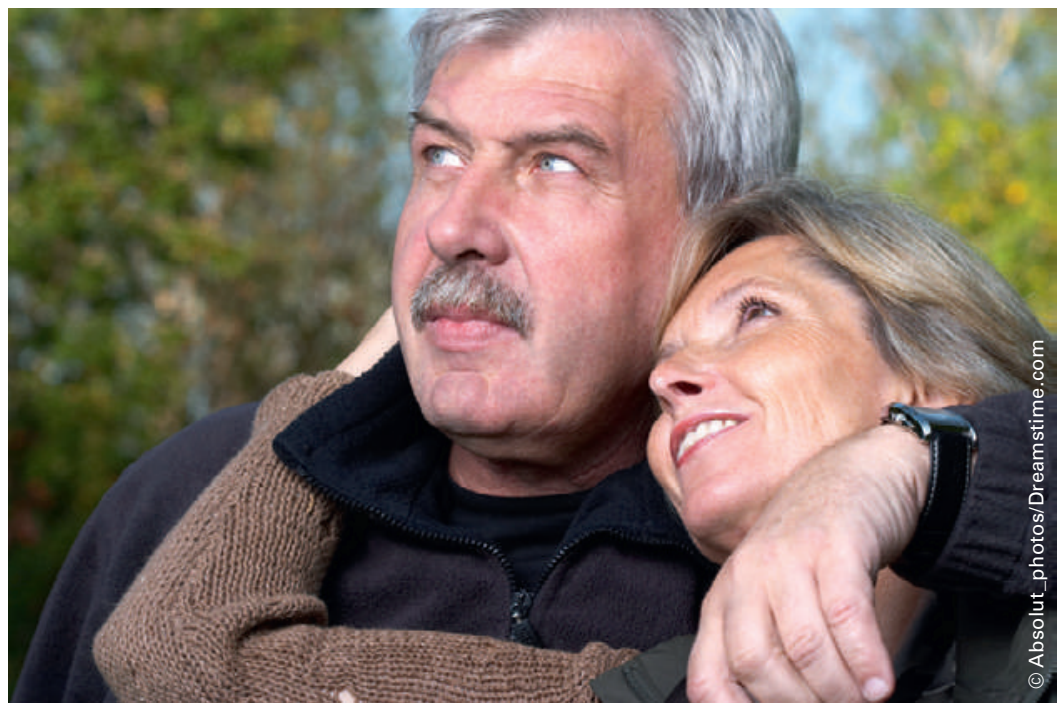

Sind alle über 50-jährigen Männer und alle über 60-jährigen Frauen an Atherosklerose erkrankt?
Korrespondenz:

Sekretariat Trägerschaft

Swiss Medical Board

Stampfenbachstrasse 30

CH-8090 Zürich

Tel. 0432592479

info[at]medical-board.ch tätsbereinigten Lebensjahren gegenüber. Darin sind die Nutzenverluste infolge Todesfälle und schwerer, jedoch nicht tödlicher kardiovaskulärer Erkrankungen eingerechnet. 2000 CHF geteilt durch 0.0095 QALY ergibt die im Bericht ausgewiesenen Kosten von 210000 CHF pro QALY beim heutigen Schwellenwert.

Die von Romanens et al. angegebene relative Risikoreduktion von $22 \%$ für die Wirksamkeit von Statinen

\section{Die relative Risikoreduktion ist nicht relevant.}

taucht im Bericht des SMB nicht auf. Die relative Risikoreduktion ist ja auch nicht relevant. Vielmehr kommt es darauf an, die Wirksamkeit und die Kosten einer $\mathrm{Be}$ handlung in absoluten Einheiten zu messen. Würde der Schwellenwert, wie vom SMB empfohlen, auf 7,5\% erhöht, so ist mit einer höheren Wirksamkeit der Statinbehandlung zu rechnen, die NNT (numbers needed to treat) wird kleiner, weil Personen mit geringerem Risiko, ein relevantes kardiovaskuläres Ereignis zu erleiden, nicht mehr behandelt würden. Wie das SMB auf Seite 25 seines Berichts schreibt, geht es für diesen Fall von einer höheren Wirksamkeit aus. Innerhalb von 5 Jahren könnten damit im Erwartungswert 2,5 Todesfälle und 11,25 nicht-tödliche Fälle vermieden werden. Dadurch würde sich der Nutzenwert auf 0.011875 QALY erhöhen und die Kosten der Statinbehandlung würden auf 1912.50 CHF pro behandelte Person sinken. Dies führt $\mathrm{zu}$ einem Kosten-Wirksamkeit-Verhältnis von rund
160000 CHF pro QALY, welches das SMB als immer noch sehr hoch einschätzt. Entgegen der Behauptung von Romanens et al. ist das Vorgehen des SMB transparent und nachvollziehbar.

Nicht nachvollziehbar sind die arithmetischen Übungen von Romanens et al. zum Thema QALY: «Die medizinischen Kosten können deshalb vernachlässigt werden [sic!], der Einfachheit halber werden 8,33 CHF pro Monat angenommen, insgesamt somit rund $100 \mathrm{CHF}$ pro Jahr [!]. Dabei ergibt sich: Bereits bei einem ESCRisiko von $2 \%$ in 10 Jahren über fünf Jahre beobachtet betragen die Kosten pro QALY nicht 210000 CHF, sondern 3365 CHF. Nach 10 Jahren zeigt sich sogar ein Netto-Gewinn pro QALY von 7791 CHF.» D.h., nach Romanens, je früher und je länger Statine verabreicht werden, desto eher resultiert ein Gewinn! Es braucht keine Arztkonsultationen, keine Laboruntersuchungen und es gibt vor allem auch keine Nebenwirkungen: Fazit: Die Statine gehören ins Trinkwasser ...

Interessant ist die Diskussion über die "Atherosklerose», die erwiesenermassen in der Bevölkerung prävalent ist, und die Frage, ob «auch kranke Arterien gesund seien». Gemäss Romanens et al. «zeigte sich bei einem ESC-Risiko von $\geq 0,9 \%$ in $76 \%$ eine Atherosklerose mit mässig hohem oder hohem Risiko und bei 18\% eine Atherosklerose mit hohem Risiko. Damit verkennt das SMB die Tatsache, dass Personen bereits mit einem ESC-Risiko von grösser als 0,9\% in 10 Jahren in 91\% eine relevante Atherosklerose haben.» Nach Ansicht von Kollege Romanens sind somit alle über 50-jährigen Männer und alle über 60-jährigen Frauen krank. Die «Definitionshoheit darüber, was gesund sei und was krank», obliegt sicher der Medizin und nicht der Ökonomie, aber einer ökonomisch verantwortungsbewussten Medizin. Die Debatte über dieses Spannungsfeld ist nicht neu: Schon 1933 schrieb Jules Romains in seinem wunderbar verfilmten Buch Dr Knock ou Le triomphe de la médecine, dass: "Tout homme bien portant est un malade qui s'ignore.» «Dass das SMB nun darüber nachdenkt, seinen Statinbericht zurückzuziehen", ist ein Wunschgedanke unserer Kritiker. Es bleibt die Aufgabe des SMB, sich für eine optimale Gesundheitsversorgung der Bevölkerung unter Berücksichtigung endlicher Ressourcen einzusetzen.

1 Romanens M, Ackermann F. SMB, AGLA und das Statin-Conundrum. Schweiz Ärztezeitung. 2014; 95(47): 1786-7.

2 Meier CA, Metzger U. Replik zum vorangegangenen Artikel «SMB, AGLA und das Statin-Conundrum». Schweiz Ärztezeitung. 2014; 95(47): 1788 